\title{
A Fractional SEIR Epidemic Model for Spatial and Temporal Spread of Measles in Metapopulations
}

\author{
Emile Franc Doungmo Goufo, ${ }^{1}$ Suares Clovis Oukouomi Noutchie, ${ }^{2}$ and Stella Mugisha ${ }^{1}$ \\ ${ }^{1}$ Department of Mathematical Sciences, University of South Africa, Florida 0003, South Africa \\ ${ }^{2}$ Department of Mathematical Sciences, North-West University, Mafikeng 2790, South Africa \\ Correspondence should be addressed to Emile Franc Doungmo Goufo; dgoufef@unisa.ac.za
}

Received 24 May 2014; Accepted 6 June 2014; Published 22 June 2014

Academic Editor: Abdon Atangana

Copyright (c) 2014 Emile Franc Doungmo Goufo et al. This is an open access article distributed under the Creative Commons Attribution License, which permits unrestricted use, distribution, and reproduction in any medium, provided the original work is properly cited.

\begin{abstract}
Measles is a higher contagious disease that can spread in a community population depending on the number of people (children) susceptible or infected and also depending on their movement in the community. In this paper we present a fractional SEIR metapopulation system modeling the spread of measles. We restrict ourselves to the dynamics between four distinct cities (patches). We prove that the fractional metapopulation model is well posed (nonnegative solutions) and we provide the condition for the stability of the disease-free equilibrium. Numerical simulations show that infection will be proportional to the size of population in each city, but the disease will die out. This is an expected result since it is well known for measles (Bartlett (1957)) that, in communities which generate insufficient new hosts, the disease will die out.
\end{abstract}

\section{Introduction and Important Facts}

Mathematical modeling in epidemiology is concerned with describing the spread of disease and its effect on people. This itself encompasses a range of disciplines, from biology, mathematics, and engineering to sociology and philosophy, all of which are utilized to a better understanding and containing of the spread of infection. The models developed in epidemiology provide a wider understanding of the mechanisms that influence the spread of a disease and they suggest control strategies [1]. One of the early triumphs of mathematical epidemiology was a formulation of a model in [2] to predict the behavior of a disease. The total population in this model was assumed to be constant and divided into three compartments: suspended, infectious, and recovered. Over the years, more complex models have been derived. Among them, we count models based on dynamics with derivative of fractional order and metapopulation models.

Fractional differential equations and their applications have been comprehensively studied in a large number of works [3-6]. Although numerous analyses have been done in modeling the dynamics of epidemiological diseases, most of them have been restricted to integer order (delay) differential equations. In recent years, it has turned out that many phenomena in different fields can be described very successfully by the models using fractional order differential equations. Adomian decomposition method has been employed to obtain solutions of a system of nonlinear fractional differential equations in number of articles and the method is extended in [5], where the authors used a complementary method, Frobenius decomposition method, to generalize the classical Darcy law. Epidemiology combined with fractional calculus was applied in [6] for an outbreak of dengue fever.

Metapopulation models are defined as system of differential equations generated by discrete spatial models with continuous time. Indeed, they describe movement of individuals between discrete spatial patches that can be cities, towns, places, and so forth. Metapopulation models have been thoroughly analyzed in numeral articles $[7,8]$.

Measles is a higher contagious viral disease caused by infection of Paramyxovirus, generally of the genus Morbillivirus. Its incubation period is located somewhere between 9 and 12 days and its infectivity period between 4 and 9 days. Measles is highly present in early childhood and its epidemics 
are commonly related to aggregation of children at schools or childcare centres. It is recommended to get vaccinated against it at around 18 months of age and have a booster at 4 to 5 years of age. The disease is particularly characterized by its low mortality and high morbidity. Measles will continue to circulate in a community with a higher number of susceptible hosts by birth of children. However, in communities which generate insufficient new hosts, measles will die out. This theory was introduced in 1957 by Bartlett [9], who brought out the critical population size for a community and referred it to be the minimum number supporting measles.

Our work is motivated by an article by the online magazine Otago Daily Time [10], following the emergence of measles after a student brought the disease from Sydney where he participated in a hip hop competition. On 1 March 2014, the online magazine Otago Daily Time released a warning over measles outbreak in New Zealand after the disease was discovered on the student. After that, many cases of the disease in different cities all over the country were noticed. The metapopulation model, presented in Section 3, is then suitable to describe and analyze the spread of the disease.

\section{Formulation of Fractional Temporal SEIR Measles Model}

We formulate here the system modeling the fractional temporal spread of measles in a human population. In this model, a population supposed constant is divided into different classes, disjoint and based on their disease status. At time $t$, $S=S(t)$ is the fraction of population representing individuals susceptible to measles, $E=E(t)$ is the fraction of population representing individuals exposed to measles, $I=I(t)$ is the fraction of population representing individuals infectious with measles, and $R=R(t)$ is the fraction of population representing individuals that recovered from measles. We assume that all recruitment is done by birth into the class of susceptible and occurs at constant birth rate $b$. The rate constant for nondisease related death is $\mu$; thus $1 / \mu$ is the average lifetime. We use the standard mass balance incidence expressions $\beta(t) S I$ to indicate successful transmission of measles due to effective contacts dynamics in the population by infectious individuals. Once infected, a fraction of exposed people becomes infectious with a constant rate $\sigma$, so that $1 / \sigma$ is the average incubation period. Some infectious individuals will recover after a treatment or a certain period of time at a rate constant $\zeta$, making $1 / \zeta$ the average infectious period. The transfer diagram for this model is described by Figure 1 and formulated by the following differential equations:

$$
\begin{aligned}
& D_{t}^{\gamma} S=b-(\beta(t) I+\mu) S, \\
& D_{t}^{\gamma} E=\beta(t) S I-(\sigma+\mu) E, \\
& D_{t}^{\gamma} I=\sigma E-(\zeta+\mu) I, \\
& D_{t}^{\gamma} R=\zeta I-\mu R,
\end{aligned}
$$

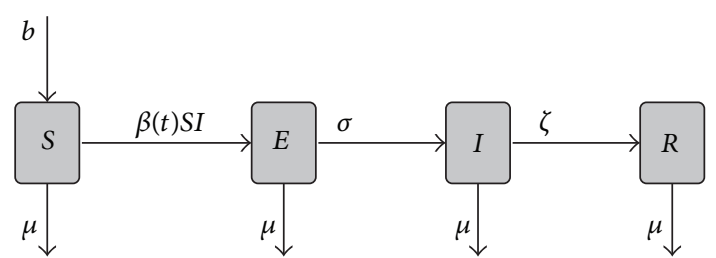

FIGURE 1: Transfer diagram for temporal dynamics transmission of measles.

where

$$
D_{t}^{\gamma} u(t)=\frac{\partial^{\gamma}}{\partial t^{\gamma}} u(t)=\frac{1}{\Gamma(1-\gamma)} \int_{0}^{t}(t-r)^{-\gamma} \frac{\partial}{\partial r} u(r) d r
$$

with $0 \leq \gamma<1$ being the fractional derivative of the function $u(t)$ in the sense of Caputo [3], with $\Gamma$ being the Gama function.

\section{Metapopulation Model}

In this section we formulate the metapopulation dynamics [7] for four patches representing four cities in New Zealand: Auckland, Bay of Plenty, Wellington, and Dunedin. Those cities are chosen since, on 1 March 2014, there were 43 cases of individuals found infected with measles in Auckland (among which 20 students were of the same school), 15 cases in the city of Bay of Plenty, and 3 cases in Wellington. There was no case found in Dunedin, a city in the southern region of Otago in South Island of New Zealand. The aim is to deal with prevention against measles to reach the region of Otago. For reasons of commodity, we denote the cities Auckland, Bay of Plenty, Wellington, and Dunedin by the letters $A, B, W$, and $D$, respectively. Let us consider the set $\mathscr{P}=\{A ; B ; W ; D\}$ of the four patches. We call $m_{x y}^{c}$ the rate of travel from city $x$ to city $y$ in compartment $c$ with $c=S, E, I$, or $R$; that is, it represents the transfer rate of individuals in the compartment $c$ of city $x$ moving to the same compartment $c$ in city $y$. It is clear that $m_{x x}^{c}=0$, for all $x \in \mathscr{P}$ and $c \in \mathscr{C}=\{S, E, I, R\}$. Then the travel model is given in Figure 2 and, for each city $x \in \mathscr{P}$, we obtain the system of four differential equations given by

$$
\begin{aligned}
D_{t}^{\gamma} S_{x}= & b_{x}-\left(\beta_{x}(t) I_{x}+\mu_{x}\right) S_{x}+\sum_{y \in \mathscr{P}} S_{y} m_{y x}^{S}-S_{x} \sum_{y \in \mathscr{P}} m_{x y}^{S} \\
D_{t}^{\gamma} E_{x}= & \beta_{x}(t) S_{x} I_{x}-\left(\sigma_{x}+\mu_{x}\right) E_{x} \\
& +\sum_{y \in \mathscr{P}} E_{y} m_{y x}^{E}-E_{x} \sum_{y \in \mathscr{P}} m_{x y}^{E}, \\
D_{t}^{\gamma} I_{x}= & \sigma_{x} E_{x}-\left(\zeta_{x}+\mu_{x}\right) I_{x}+\sum_{y \in \mathscr{P}} I_{y} m_{y x}^{I}-I_{x} \sum_{y \in \mathscr{P}} m_{x y}^{I}, \\
D_{t}^{\gamma} R_{x}= & \zeta_{x} I_{x}-\mu_{x} R_{x}+\sum_{y \in \mathscr{P}} R_{y} m_{y x}^{R}-R_{x} \sum_{y \in \mathscr{P}} m_{x y}^{R},
\end{aligned}
$$


TABLE 1

\begin{tabular}{lcccc}
\hline & Auckland & Bay of Plenty & Wellington & Dunedin \\
Initial population $\left(\times 10^{5}\right)$ & $S_{A}(0)=1417,9$ & $S_{B}(0)=124,1$ & $S_{W}(0)=397,5$ & $S_{D}(0)=118,25$ \\
\hline Auckland & 0 & & & \\
Bay of Plenty & 200 & 0 & 0 & 0 \\
Wellington & 650 & 470 & 610 & 0 \\
Dunedin & 1060 & 1020 & & \\
\hline
\end{tabular}

Approximate distances between cities (km).

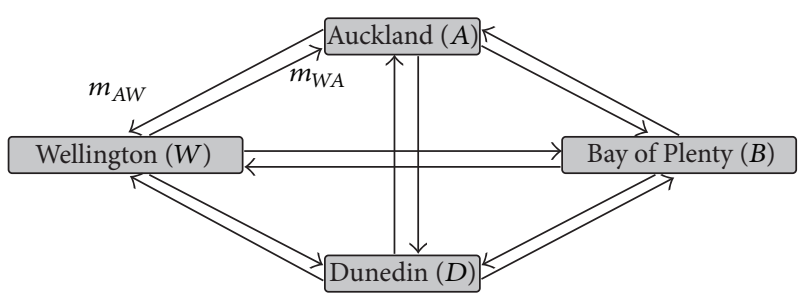

Figure 2: Travel model dynamics between cities. The arrows symbolize the travel rates $m_{x y}$ between cities $x$ and $y$.

with initial conditions

$$
\begin{aligned}
& S_{x}(0)>0, \quad E_{x}(0) \geq 0, \quad I_{x}(0) \geq 0, \quad R_{x}(0) \geq 0, \\
& \text { with } \sum_{x \in \mathscr{P}} E_{x}(0)+I_{x}(0) \geq 0 .
\end{aligned}
$$

These initial conditions ensure that, at the beginning, there are infected individuals in the system. They also imply the well posedness of the model. Indeed, we have the generalized version of the Mean Value Theorem that is stated as follows.

Theorem 1. Let the function $\mathcal{g} \in C\left[t_{1}, t_{2}\right]$ and its fractional derivative $D_{t}^{\alpha} \mathcal{J} \in C\left(t_{1}, t_{2}\right]$ for $0 \leq \alpha<1$, and $t_{1}, t_{2} \in \mathbb{R}$; then one has

$$
\mathscr{J}(t)=\mathscr{J}\left(t_{1}\right)+\frac{1}{\Gamma(\alpha)} D_{t}^{\alpha} \mathscr{J}(\tau)\left(t-t_{1}\right)^{\alpha} \quad \forall t \in\left(t_{1}, t_{2}\right]
$$

where $0 \leq \tau<t$.

Proof. See the "Generalized Mean Value Theorem" proved in [11].

Thus, consider the interval $\left[0, t_{2}\right]$ for any $t_{2}>o$; this theorem implies that the function $\mathscr{J}:\left[0, t_{2}\right] \rightarrow \mathbb{R}^{+}$is nonincreasing on $\left[0, t_{2}\right]$ if $D_{t}^{\alpha} \mathcal{F}(t) \leq 0$ for all $t \in\left(0, t_{2}\right)$ and nondecreasing on $\left[0, t_{2}\right]$ if $D_{t}^{\alpha} \mathscr{J}(t) \geq 0$ for all $t \in\left(0, t_{2}\right)$. Furthermore, the nonnegativity of solutions to the problem (3)-(4) is obvious since the total population in city $x$, say $N_{x}$, evolves according to the sum of the four equations in (3) such that a positive $N_{x}$ yields nonnegative solutions of (3)-(4).

\section{Equilibrium Points, Basic Reproduction Ratio, and Stability}

We evaluate the equilibrium points of (3) by letting, for each city $x \in \mathscr{P}$,

$$
\begin{aligned}
& D_{t}^{\gamma} S_{x}=0, \\
& D_{t}^{\gamma} E_{x}=0, \\
& D_{t}^{\gamma} I_{x}=0, \\
& D_{t}^{\gamma} R_{x}=0 .
\end{aligned}
$$

The disease-free equilibrium (DFE) of the system is obtained by setting $E_{x}(0)=0, I_{x}(0)=0$ for each city $x \in \mathscr{P}$ and its stability is investigated using the next generation operator [12]. Thus, at the DFE for all the cities $x \in \mathscr{P}$, we have $S_{x}=N_{x}$ and adding the equation given in (3) yields at the DFE

$$
b_{x}-\mu_{x} N_{x}+\sum_{y \in \mathscr{P}} N_{y} m_{y x}^{S}-\sum_{y \in \mathscr{P}} N_{x} m_{x y}^{S}=0 .
$$

Following the same approach as [7], we compute the basic reproduction number as the spectral radius

$$
\mathscr{R}_{0}=\rho\left(F V^{-1}\right),
$$

where $F$ and $V$ are, respectively, the matrix of new infections and the matrix of transfer between compartments. The disease-free equilibrium of system (3) is linearly stable if $\mathscr{R}_{0}<1$ and unstable if $\mathscr{R}_{0}>1$.

\section{Numerical Simulations}

To provide numerical approximations and simulations of solutions to the fractional model (3)-(4) with different values of $\gamma$, we use the implementation code of the predictorcorrector PECE method of Adams-Bashforth-Moulton type described in [13]. We restrict our analysis to the case of symmetry in the intercities movements, meaning $m_{x y}=m_{y x}$. We assume that $m_{x y}$ is inversely proportional to the distance $D_{x y}$ between the cities $x$ and $y$. That is,

$$
m_{x y} D_{x y}=k
$$

for some $k \in \mathbb{R}$. Table 1 shows the different distances between cities. Figure 3 shows the dynamics of the disease in the city of Auckland $(A)$, for the four compartments with different 

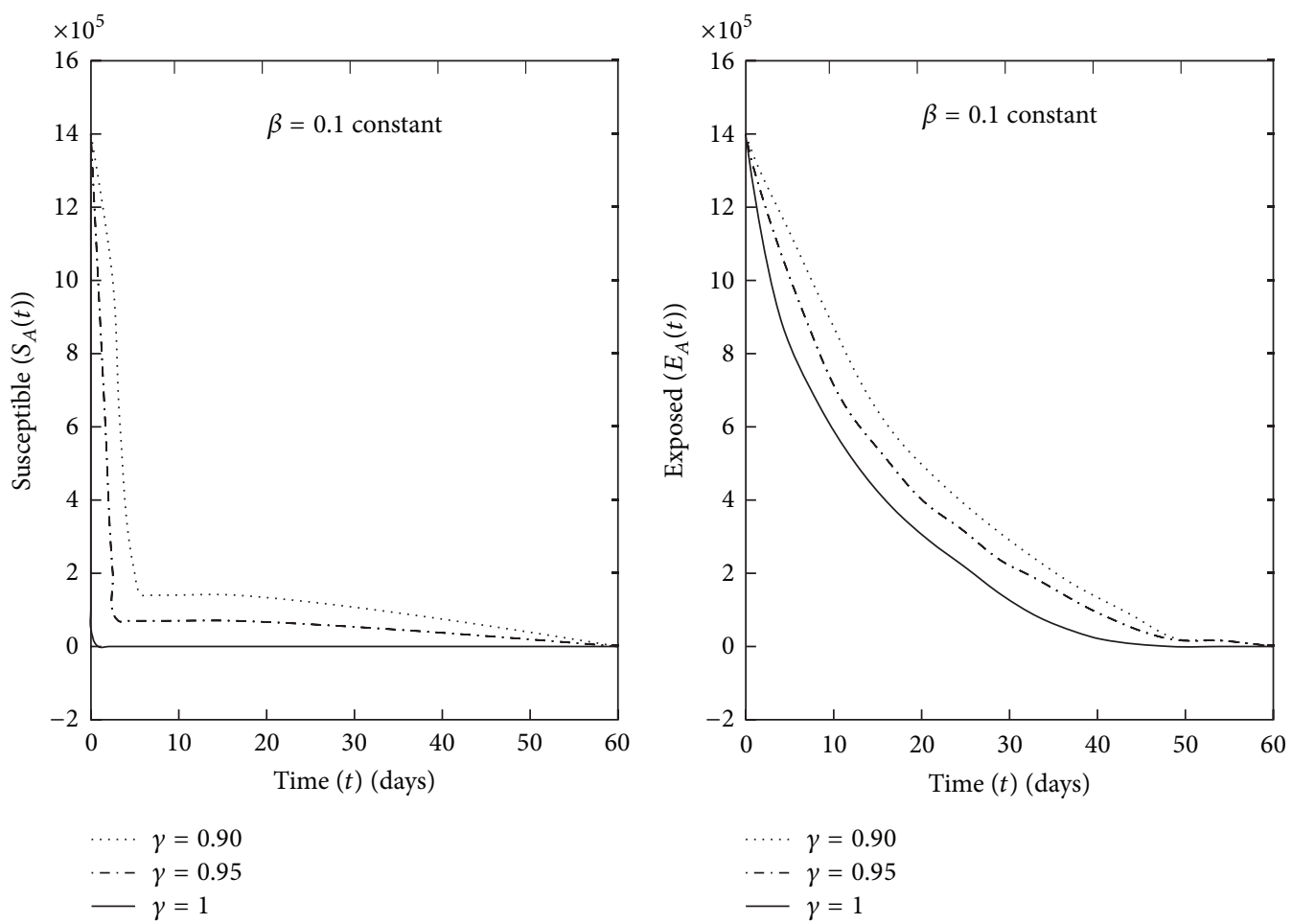

(a)

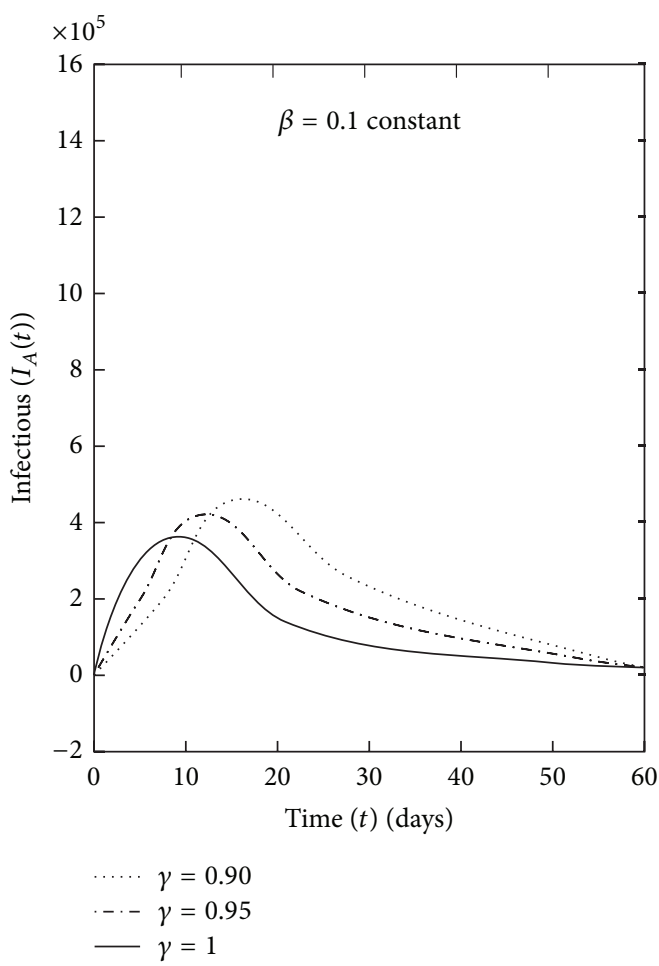

(c)

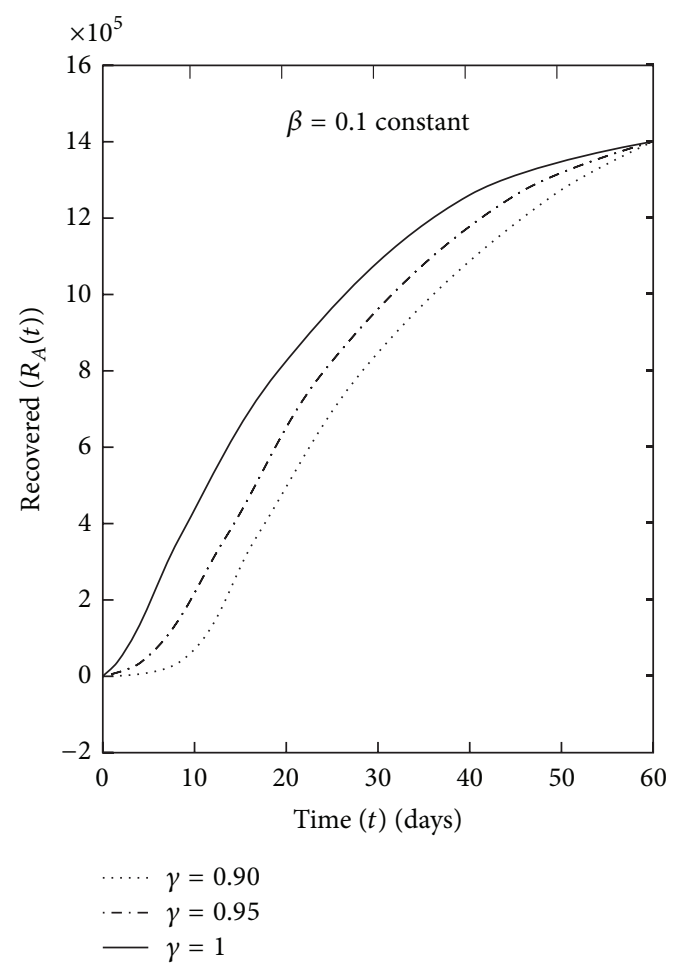

(d)

Figure 3: Measles dynamics in Auckland $(A)$ for the 4 compartments for $\gamma=1 ; 0.95 ; 0.90$. 

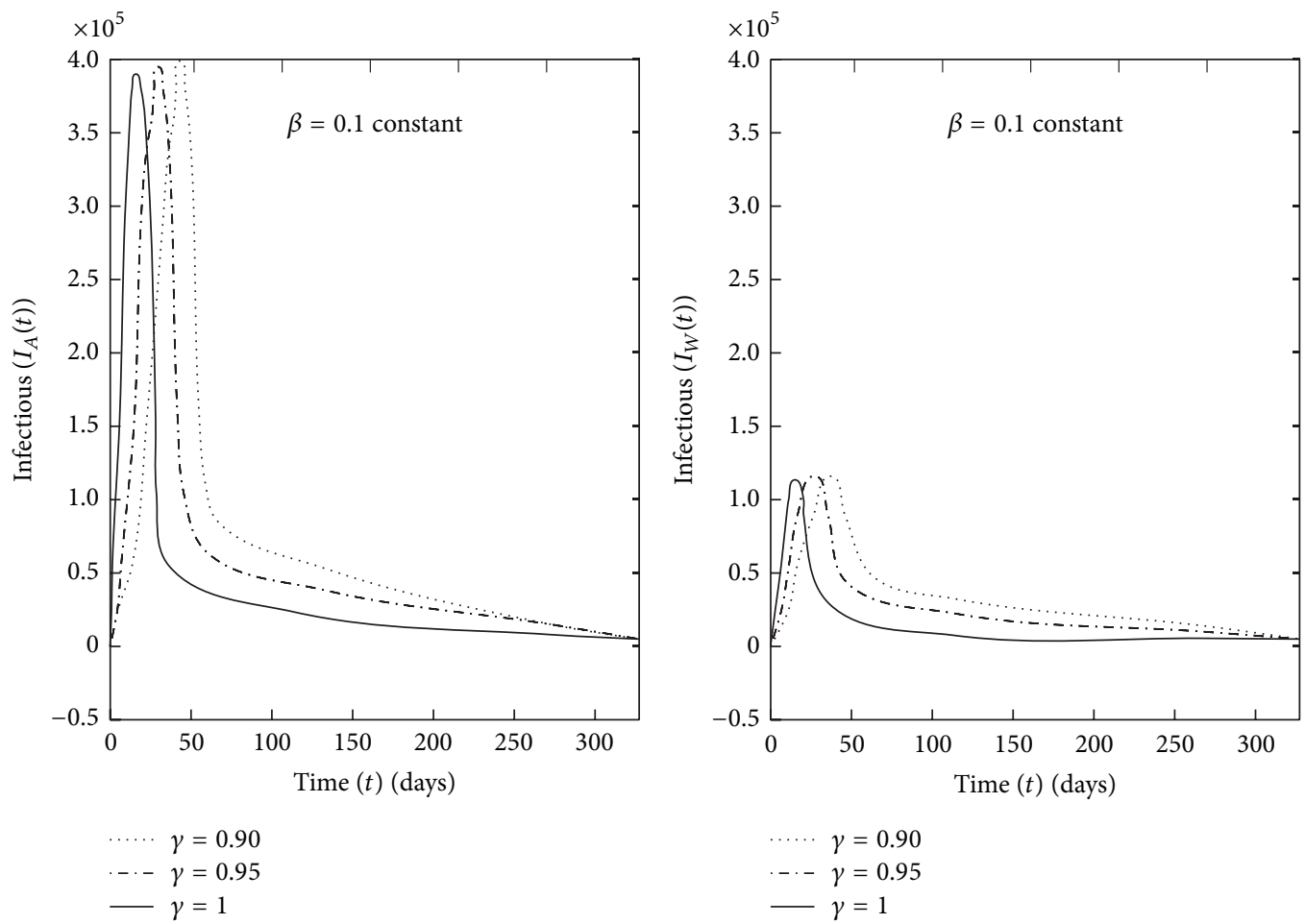

(a)

(b)

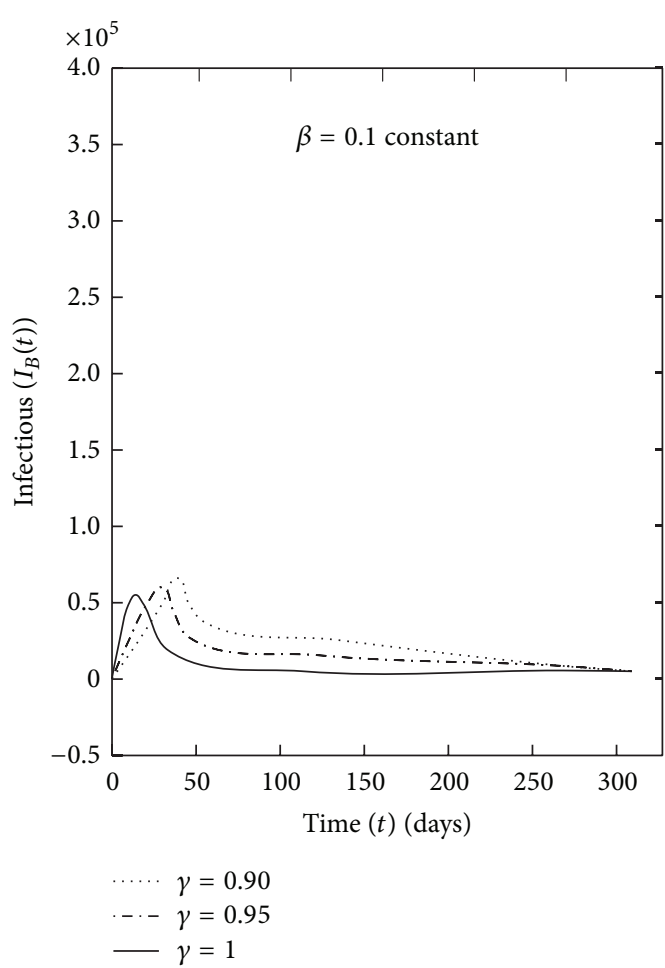

(c)

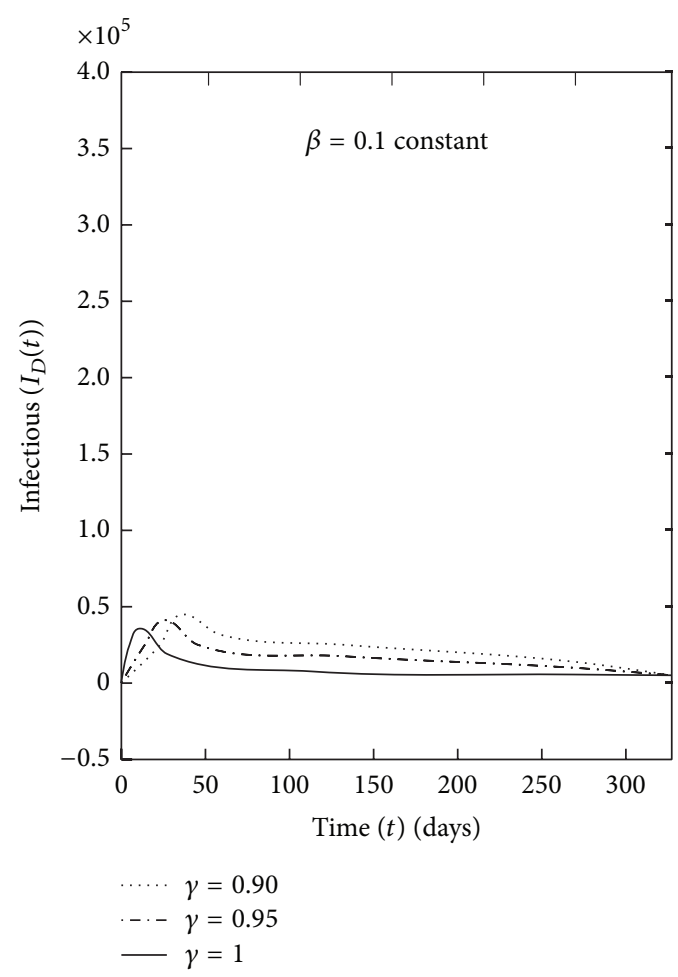

(d)

FIgURE 4: Global dynamics of the infection in the 4 cities for $\gamma=1 ; 0.95 ; 0.90$. 
values of the fractional derivative order $\gamma=1 ; 0.95$ and 0.90 . Auckland has been chosen just as an example since it contains the highest number of people among the four cities as shown in Table 1. In Figure 4, we can see the global evolution the infection in the four cities, with different values of $\gamma$. It is clear that the disease dies out irrespective of the three values of $\gamma$. In the simulation for the city $x=A$ (Auckland), for example, we have taken $E_{A}(0)=1, I_{A}(0)=0.01$, and $R_{A}(0)=0.02$ and have considered the parameters

$$
\begin{aligned}
& \zeta_{A}=0.14, \\
& \mu_{A}=0, \\
& \beta_{A}=0.1, \\
& k=1, \\
& \sigma_{A}=0.09, \\
& b_{A}=0 .
\end{aligned}
$$

\section{Concluding Remarks}

In this paper we have presented the well posedness of fractional SEIR metapopulation dynamics of measles between four cities in New Zealand. We used real data according to the article by the online magazine Otago Daily Time, following the emergence of the disease after a student brought it from Sydney where he participated in a hip hop competition. We have started by showing nonnegativity of solutions to the fractional metapopulation model, thereby addressing the problem of its well posedness. We have also shown that the disease-free equilibrium of the model is linearly stable if $\mathscr{R}_{0}<1$ and unstable if $\mathscr{R}_{0}>1$. Numerical simulations have shown that, even in fractional dynamics of measles in metapopulation, the epidemic will not occur in communities which generate insufficient new hosts, which is in accordance with the theory of Bartlett [9]. This work generalizes the preceding ones with the inclusion of the fractional dynamics to a combined SEIR and metapopulation model, giving at the same time one of the multiple applications of fractional differential equations.

\section{Conflict of Interests}

The authors declare that there is no conflict of interests regarding the publication of this paper.

\section{References}

[1] F. Brauer and C. Castillo-Chávez, Mathematical Models in Population Biology and Epidemiology, vol. 40 of Texts in Applied Mathematics, Springer, New York, NY, USA, 2001.

[2] W. O. Kermack and A. G. McKendrick, "A contribution to mathematical theory of epidemics," Proceedings of the Royal Society A, vol. 115, pp. 700-721, 1927.

[3] M. Caputo, "Linear models of dissipation whose Q is almost frequency independent," Journal of the Royal Australian Historical Society, vol. 13, part 2, pp. 529-539, 1967.

[4] A. Atangana and E. Alabaraoye, "Solving system of fractional partial differential equations arisen in the model of HIV infection of $\mathrm{CD}^{+}$cells and attractor one-dimensional KellerSegel equation," Advances in Difference Equations, vol. 2013, article 94, 2013.

[5] A. Atangana and P. D. Vermeulen, "Analytical solutions of a space-time fractional derivative of groundwater flow equation," Abstract and Applied Analysis, vol. 2014, Article ID 381753, 11 pages, 2014.

[6] K. Diethelm, "A fractional calculus based model for the simulation of an outbreak of dengue fever," Nonlinear Dynamics, vol. 71, no. 4, pp. 613-619, 2013.

[7] J. Arino and P. van den Driessche, "Metapopulation epidemic models. A survey," Fields Institute Communications, vol. 48, pp. 1-13, 2006.

[8] L. A. Rvachev and I. M. Longini Jr., "A mathematical model for the global spread of influenza," Mathematical Biosciences, vol. 75, no. 1, pp. 3-22, 1985.

[9] M. S. Bartlett, "Measles periodicity and community size," Journal of the Royal Statistical Society A, no. 120, pp. 48-70, 1957.

[10] Otago Daily Time, March 2014, http://www.odt.co.nz/.

[11] Z. M. Odibat and N. T. Shawagfeh, "Generalized Taylor's formula," Applied Mathematics and Computation, vol. 186, no. 1, pp. 286-293, 2007.

[12] P. van den Driessche and J. Watmough, "Reproduction numbers and sub-threshold endemic equilibria for compartmental models of disease transmission," Mathematical Biosciences, vol. 180, pp. 29-48, 2002.

[13] K. Diethelm, N. J. Ford, A. D. Freed, and Yu. Luchko, "Algorithms for the fractional calculus: a selection of numerical methods," Computer Methods in Applied Mechanics and Engineering, vol. 194, no. 6-8, pp. 743-773, 2005. 


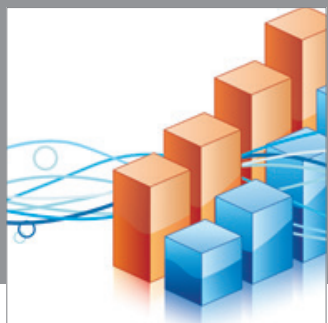

Advances in

Operations Research

mansans

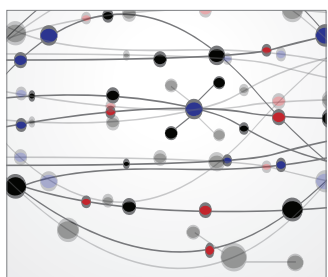

The Scientific World Journal
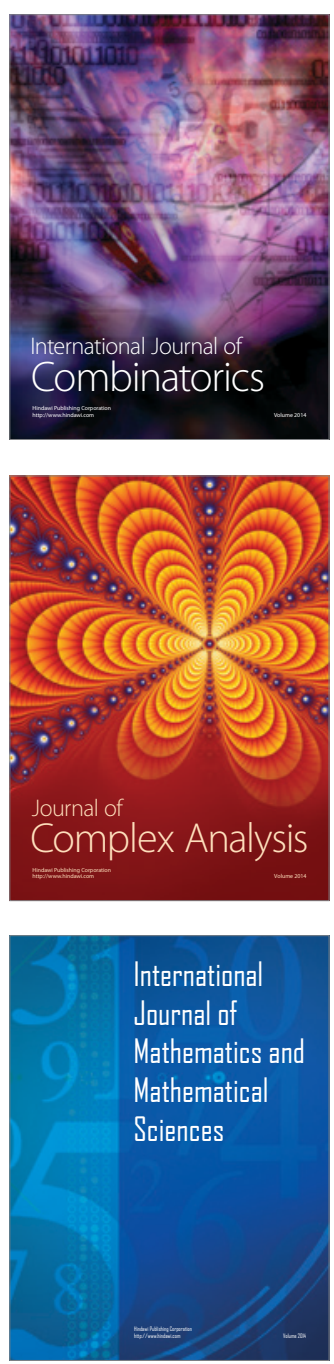
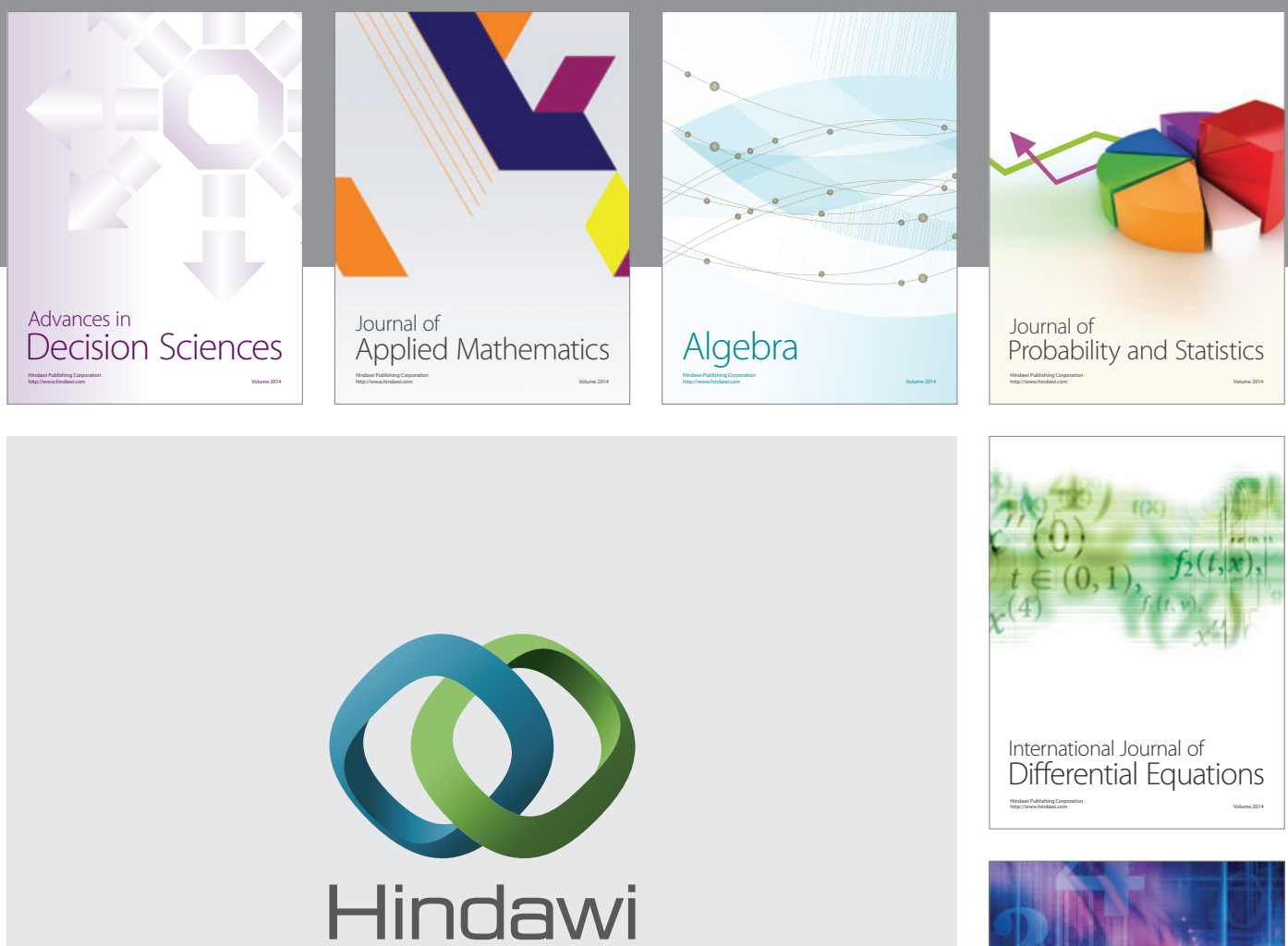

Submit your manuscripts at http://www.hindawi.com
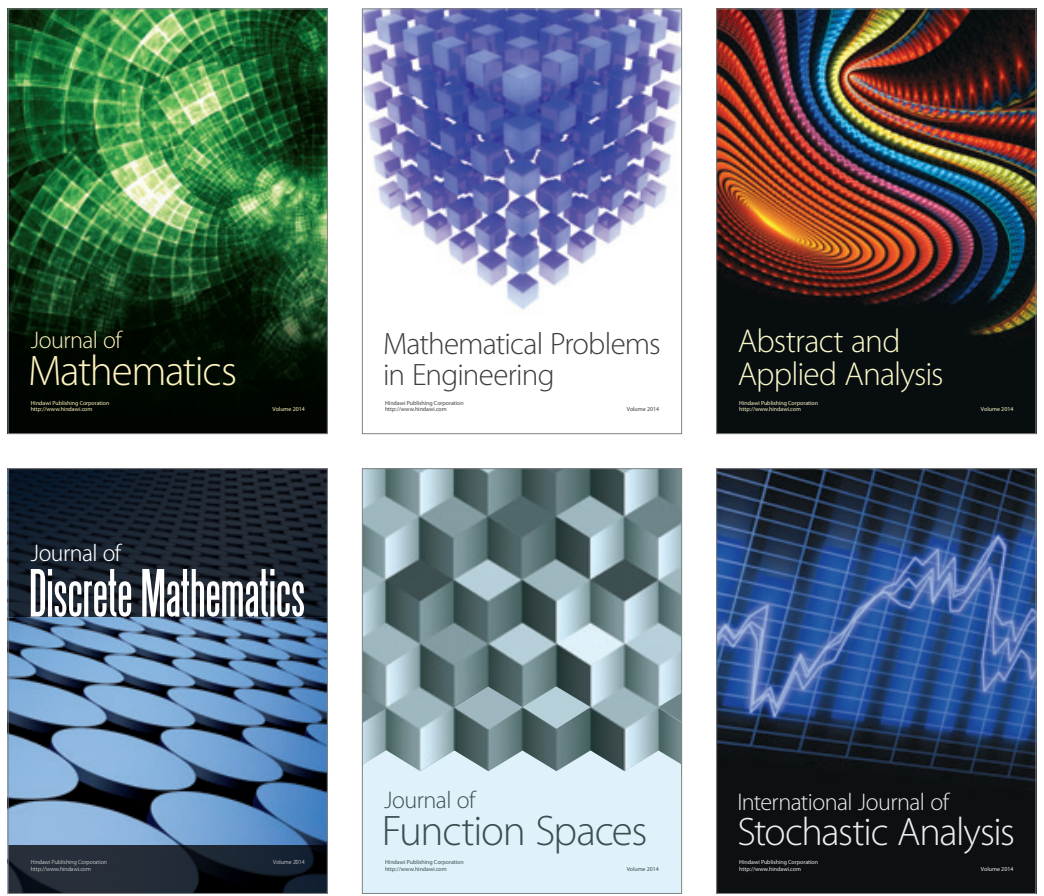

Journal of

Function Spaces

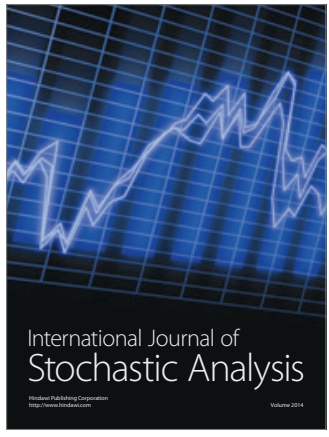

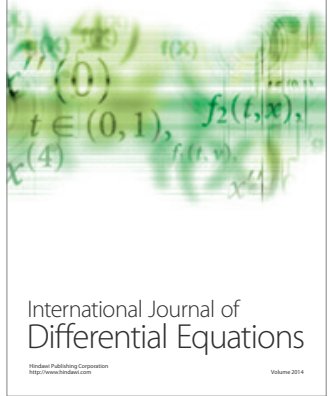
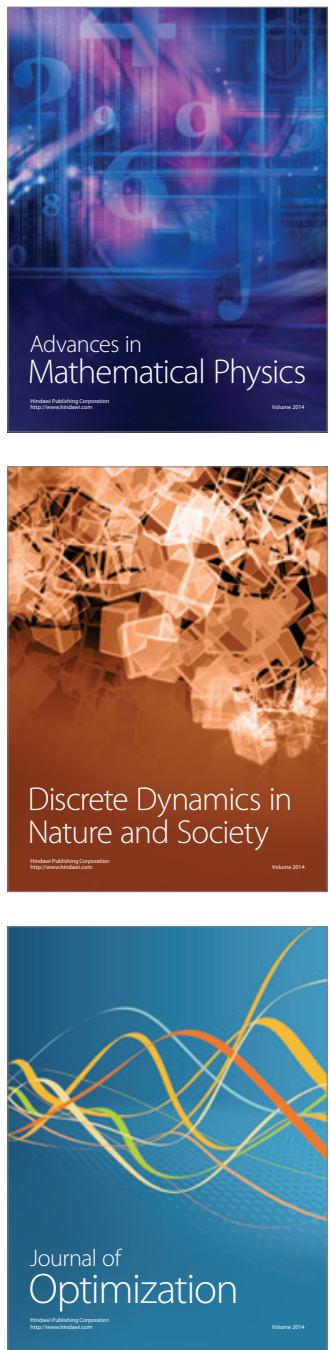